\title{
Soutenir le transfert des connaissances pour les interventions en matière de basse vision grâce à un répertoire en ligne consacré à des outils d'évaluation validés
}

\author{
Walter Wittich, \\ PhD FAAO CLVT \\ Professeur adjoint, \\ École d'optométrie \\ Université de Montréal
}

Catherine Houtekier, M.B.S.I. Agente de planification, de programmation et de recherche CRIR/Institut Nazareth et Louis-Braille du CISSS de la Montérégie-Centre

Sylvie Cantin, M.A. Agente de planification, de programmation et de recherche CRIR/Institut Nazareth et Louis-Braille du CISSS de la Montérégie-Centre

\section{Lettre de recherche}

Le choix des mesures de résultats pour les études cliniques ou de population à grande échelle au Canada contenant des évaluations objectives et subjectives de la fonction visuelle, comme l'Étude longitudinale canadienne sur le vieillissement $(E L C V)^{1}$ ou l'Étude d'évaluation globale de la neurodégénérescence et de la démence (COMPASS-ND) ${ }^{2}$, est limité par la disponibilité d'outils d'évaluation validés dans les deux langues officielles. De même, les professionnels de la santé visuelle n'ont pas le temps de chercher dans la littérature de recherche des mesures de résultats validées qu'ils peuvent utiliser avec des patients anglophones ou francophones, ce qui rend difficile toute comparaison significative. Cette difficulté est encore plus prononcée dans les sous-spécialités cliniques telles que la basse vision. Par ailleurs, les chercheurs orientent principalement leurs publications vers un public universitaire, avec de rares exemples présentant des découvertes récentes dans un format prêt à être utilisé dans l'environnement clinique ${ }^{3}$.

Afin de combler ce fossé, nous présentons un répertoire en ligne qui encourage l'adoption d'outils de recherche validés dans la pratique clinique, appropriés pour les personnes présentant une déficience visuelle, et qui sont disponibles en français, ou en français et en anglais. ORVIS (Outils pour la Réadaptation de la VISion / DirectORy of Tools for VISion Rehabilitation, www.orvis.vision) est une base de données sur des outils de mesure dont la validité et la fidélité ont été ou sont en cours d'évaluation [4]. Son but est de faciliter les activités de transfert de connaissances en intégrant les outils de recherche dans la pratique clinique et de répondre à un besoin clinique. Ces outils favorisent l'évaluation rigoureuse et fiable des caractéristiques pertinentes pour l'évaluation, le traitement et la réadaptation des personnes ayant des déficiences visuelles, et comprennent des instruments d'évaluation fonctionnelle, perceptuelle, cognitive et psychologique. Chaque outil est présenté dans une fiche descriptive qui identifie l'outil, ses caractéristiques métrologiques, et qui liste les références bibliographiques des études scientifiques consultées. L'équipe de gestion du projet comprend un chercheur, responsable de l'infrastructure (financée en partie par le Réseau de recherche en santé de la vision FRQ-S : http://visionnetwork.ca/), deux professionnelles de recherche, une professionnelle de l'information et une technicienne en documentation de l'Institut Nazareth et Louis-Braille du CISSS de la Montérégie-Centre. Deux cliniciens de l'Institut s'ajoutent à l'équipe de projet pour former un comité consultatif afin d'assurer un lien continu et pertinent entre la recherche et la pratique clinique.

En date de décembre 2019, 29 fiches descriptives d'outils sont disponibles gratuitement. Les statistiques de consultation reflètent l'intérêt croissant suscité par le répertoire (voir figure 1). Entre sa mise en ligne en novembre 2015 et le 31 décembre 2019, www.orvis.vision a été visité 16867 fois par 
10319 visiteurs uniques de plus de 15 pays, dont la plupart font partie de la Francophonie (voir figure 2). Une adresse courriel dédiée permet aux internautes d'envoyer des demandes d'information directement à l'équipe de projet. À la lumière de ces résultats, www.orvis.vision entend poursuivre ses travaux en se concentrant sur trois objectifs : 1) continuer de choisir des outils à documenter dans l'optique d'un transfert de connaissances dans la recherche clinique en réadaptation de la basse vision; 2) produire des traductions françaises validées de certains tests ou échelles de mesure pertinents actuellement disponibles en anglais seulement; et 3) promouvoir plus largement le répertoire. En fin de compte, l'équipe ORVIS vise à renforcer la pratique de la réadaptation visuelle fondée sur des données probantes tout en favorisant la recherche intégrée qui inclut les participants et les usagers dans les deux langues officielles.

Figure 1

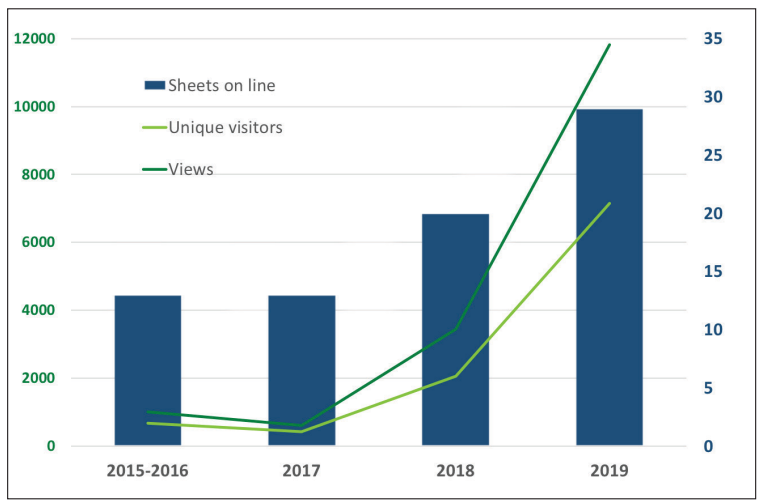

Figure 2

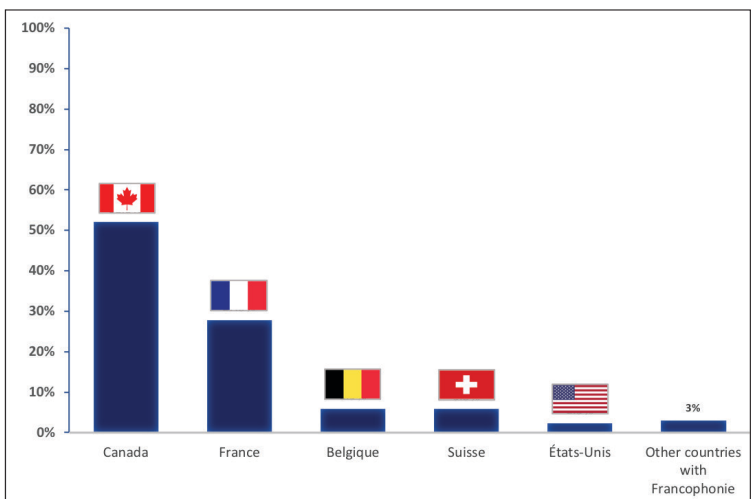

DÉCLARATION DES CRÉDITS DE L'AUTEUR :

Walter Wittich : Conceptualisation, Méthodologie, Enquête, Rédaction - Ébauche originale, Supervision, Acquisition de fonds ; Sylvie Cantin : Conceptualisation, Méthodologie, Validation, Investigation, Analyse formelle, Ressources, Conservation des données, Rédaction - Révision et édition, Administration du projet ; Catherine Houtekier : Conceptualisation, Méthodologie, Validation, Analyse formelle, Investigation, Ressources, Conservation des données, Rédaction - Révision et édition, Visualisation, Administration du projet.

MOTS CLÉS :

basse vision, réadaptation, évaluation

AUTEUR-RESSOURCE :

Walter Wittich, walter.wittich@umontreal.ca

\section{REMERCIEMENTS :}

Ces travaux sont financés par une subvention d'infrastructure du Réseau de recherche en santé de la vision du Fonds de recherche du Québec - Santé (FRQS) et soutenus par la Fondation EnVue de l'Institut Nazareth et Louis Braille du CISSS de la Montérégie Centre.

\section{REFERENCES}

1. Raina P, Wolfson C, Kirkland SA, et al. The Canadian Longitudinal Study on Aging (CLSA). Can J Aging 2009;28:221-9. https://doi. org/10.1017/S0714980809990055.

2. Chertkow H, Borrie M, Whitehead V, et al. The comprehensive assessment of neurodegeneration and dementia: Canadian cohort study. Can J Neurol Sci 2019;46:499-511. https://doi.org/10.1017/ cjn.2019.27.
3. Markowitz SN. State-of-the-art: low vision rehabilitation. Can J Ophthalmol / J Can d'Ophtalmologie 2016;51:59-66. https://doi. org/10.1016/j.jcjo.2015.11.002.

4. Wittich W. ORVIS: a directory of tools for vision rehabilitation. Ann Eye Sci 2018;3:AB106. 